\title{
Application of Glass Fiber-Based N-Doped Titania under Visible-Light Exposure for Photocatalytic Degradation of Aromatic Pollutants
}

\author{
Wan-Kuen Jo, ${ }^{1}$ Seung-Ho Shin, ${ }^{1}$ and Ho-Hwan Chun ${ }^{2}$ \\ ${ }^{1}$ Department of Environmental Engineering, Kyungpook National University, Daegu 702-701, Republic of Korea \\ ${ }^{2}$ Department of Naval Architecture and Ocean Engineering, Pusan National University, 63 Jangjeon-dong, Geumjeong-gu, \\ Busan 609-735, Republic of Korea \\ Correspondence should be addressed to Wan-Kuen Jo; wkjo@knu.ac.kr
}

Received 5 February 2014; Revised 13 March 2014; Accepted 13 March 2014; Published 31 March 2014

Academic Editor: Jiaguo Yu

Copyright (C) 2014 Wan-Kuen Jo et al. This is an open access article distributed under the Creative Commons Attribution License, which permits unrestricted use, distribution, and reproduction in any medium, provided the original work is properly cited.

Flexible glass fiber-supported nitrogen-doped titanium dioxide $\left(\mathrm{GF}-\mathrm{N}-\mathrm{TiO}_{2}\right)$ photocatalysts with different $\mathrm{N} / \mathrm{Ti}$ ratio were prepared using a dip-coating method followed by a low-temperature heat-treatment process. In addition, their photocatalytic activities were evaluated for the degradation of aromatic volatile organic compounds (VOCs) under visible-light irradiation. The prepared GF-N-TiO 2 photocatalysts were characterized using scanning electron microscopy, energy-dispersive X-ray spectroscopy, X-ray diffraction spectroscopy, and UV-visible spectroscopy. A control photolysis test performed using an uncoated GF displayed no detectable degradation of the target compounds under visible-light irradiation. The outlet-to-inlet concentration ratios of the target pollutants obtained using the GF-N-TiO 2 photocatalysts were lower than that obtained using the $\mathrm{GF}-\mathrm{TiO}_{2}$ photocatalyst. The photocatalytic activity of $\mathrm{GF}-\mathrm{N}-\mathrm{TiO}_{2}$ photocatalyst increased as the N-to-Ti ratio increased from 0.06 to 0.08 but decreased gradually as the $\mathrm{N}$-to-Ti ratio increased further to 0.12 , suggesting the existence of optimal N/Ti ratios. The outlet-to-inlet concentration ratio of all the target compounds displayed an increasing trend as both air flow rate and inlet concentration increased. Overall, the GF-N-TiO 2 photocatalysts could be applied effectively for the degradation of aromatic VOCs under visible-light irradiation when operation conditions are optimized.

\section{Introduction}

A significant amount of concern has been directed to volatile organic compounds (VOCs) due to not only their widespread presence in various environments but also their environmental health hazards. Particularly, monocyclic aromatic VOCs, including toluene, ethyl benzene, and xylene (TEX), which originate mainly from traffic emissions in urban environments, industrial combustion processes, solvent and petroleum handling, and other industrial processes, are frequently measured at high concentration levels [1]. Ambientair TEX can infiltrate buildings, exacerbating indoor air pollution [2]. Indoor air quality can be worsened due to TEX emissions from various indoor sources, such as building finishing materials, furniture, and household products [3, 4]. Moreover, TEX even at sub-ppm concentrations are potentially toxic to human beings [5], necessitating the development of control tools to lower health risks to building occupants from indoor air exposure.

Titanium dioxide $\left(\mathrm{TiO}_{2}\right)$ is a representative semiconductor photocatalyst that has been extensively used for environmental pollutant purifications. However, it has a major drawback in its practical application in that it is functional under only UV exposure conditions because of its wide band gap [6]. To address this drawback of $\mathrm{TiO}_{2}$, several strategies have been applied by a great deal of research groups; these strategies have included nonmetal deposition $[7,8]$, metal deposition $[9,10]$, dye sensitization [11], semiconductor coupling [12], and carbon materials coupling [13, 14]. Among them, nonmetal $\mathrm{N}$-doping has received special attention for environmental applications, mainly because $\mathrm{N}$ can be easily incorporated into two different sites of the 
bulk phase of $\mathrm{TiO}_{2}$ owing to its atomic size comparable with oxygen and small ionization energy $[15,16]$. Even though there are certain questions that remain to be answered to understand the photocatalytic mechanism and behavior of Ndoped $\mathrm{TiO}_{2}\left(\mathrm{~N}-\mathrm{TiO}_{2}\right)$ under visible-light exposure [17-20], several research groups found that $\mathrm{N}-\mathrm{TiO}_{2}$ photocatalysts had superior photocatalytic activity to unmodified $\mathrm{TiO}_{2}$ under visible-light irradiation for decomposition of a variety of gaseous and aqueous environmental pollutants $[8,15,21,22]$.

Application of nanomaterials for environmental treatments necessitates a supporting substrate to minimize the separation of suspended powders in aqueous media or their uncontrolled dispersion in treated air, problems encountered in water, and air applications, respectively $[23,24]$. A range of substrates as a nanomaterial support have been investigated for the photocatalytic degradation of environmental pollutants, including activated carbon powders [25], activated carbon fiber [26, 27], glass beads [28, 29], glass tubes [21], and polymer materials [30,31]. Among these substrates, glass fiber has received particular attention primarily because of its flexibility, which enables the materials to be fitted in various geometrical-shape photocatalytic reactors [32, 33]. Moreover, Panniello et al. [32] reported that glass fibersupported $\mathrm{Fe}$-doped $\mathrm{TiO}_{2}$ prepared by a combined hydrolysis and dip-coating method displayed superior photocatalytic performance to a reference P25 TiO 2 for degradation of aqueous orange dye. However, the feasibility of other types of photocatalysts, including $\mathrm{N}-\mathrm{TiO}_{2}$, which are supported by glass fiber for the treatment of environmental pollutants, is another recent topic of research that requires further examination. Consequently, in this study, the feasibility of applying glass fiber-supported $\mathrm{N}-\mathrm{TiO}_{2}\left(\mathrm{GF}-\mathrm{N}-\mathrm{TiO}_{2}\right)$ under visible-light exposure to degradation of TEX at sub-ppm concentrations typically associated with indoor air quality issues was investigated.

\section{Experimental}

2.1. Preparation and Characterization of GF-N-TiO ${ }_{2}$ and GF$\mathrm{TiO}_{2}$. GF $(11.5 \times 22.0 \mathrm{~cm}$, Hyundai Fiber $)$ was thermally pretreated and then cleaned using methanol and distilled water. The GF was heated at $350^{\circ} \mathrm{C}$ for $4 \mathrm{~h}$ in an electric furnace to burn away organic residues on the GF surface. The pretreated GF was washed sequentially with 2-propanol, acetone, and distilled water, after which it was dried at $125^{\circ} \mathrm{C}$ for $2 \mathrm{~h}$ in a dry oven. The dried GF was further treated with $\mathrm{NaOH}$ (98\%, Sigma-Aldrich) and distilled water and then dried at $125^{\circ} \mathrm{C}$ for $2 \mathrm{~h}$ in the dry oven.

$\mathrm{N}-\mathrm{TiO}_{2}$ powder was prepared using commercially available $\mathrm{P} 25 \mathrm{TiO}_{2}$ powder and urea as a $\mathrm{N}$ source. Briefly, $10 \mathrm{~g}$ of $\mathrm{TiO}_{2}$ powder was added to specified amounts of urea solution (0.7 M, Sigma-Aldrich) in a Pyrex flask and then rigorously stirred for $2 \mathrm{~h}$ under room temperature conditions. Urea solutions of $19,25,31$, and $37 \mathrm{~mL}$ were used to synthesize $\mathrm{N}^{-\mathrm{TiO}_{2}}$ powders having $\mathrm{N}$-to-Ti ratios of $0.06,0.08,0.10$, and 0.12 , respectively. The flask was wrapped with aluminum foil to prevent transmission gain of light from laboratory fluorescent lamps and then kept for $24 \mathrm{~h}$. Subsequently, the mixture in the flask was dried for $48 \mathrm{~h}$ under reduced pressure conditions to

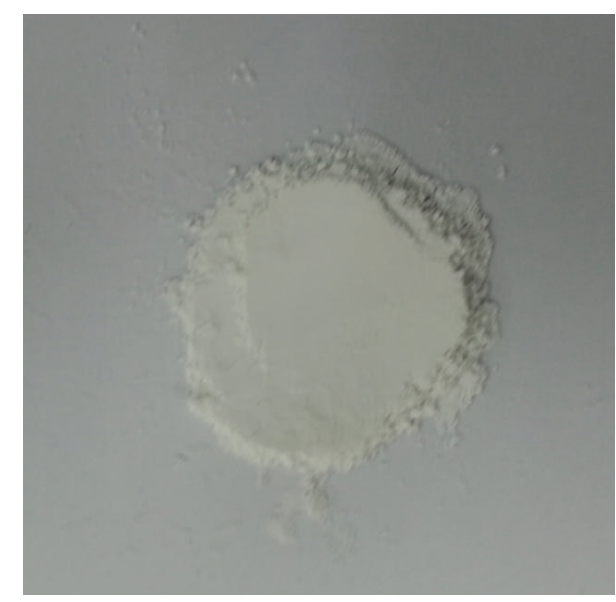

FIGURE 1: Photograph of as-prepared $\mathrm{N}-\mathrm{TiO}_{2}$ powder.

give a white powder. This powder was calcined at $550^{\circ} \mathrm{C}$ for $5 \mathrm{~h}$ in an electric furnace, after which it was washed with diluted $\mathrm{H}_{2} \mathrm{SO}_{4}$ (10\%, Sigma-Aldrich), and then distilled water until the $\mathrm{pH}$ value of washed water reached 7 . The washed powder was vacuum-dried for $12 \mathrm{~h}$ and then pulverized using an agate mortar to obtain $\mathrm{N}-\mathrm{TiO}_{2}$ powder (Figure 1).

The $\mathrm{GF}-\mathrm{N}-\mathrm{TiO}_{2}$ photocatalyst was prepared using a dip-coating method followed by a low-temperature heattreatment process. Specifically, $50 \mathrm{~mL}$ titanium (IV) isopropoxide (TTIP, 97\%, Sigma-Aldrich) was added to $10 \mathrm{~mL}$ glacial acetic acid (99\%, Sigma-Aldrich) with stirring. This solution was mixed with $1000 \mathrm{~mL}$ distilled water followed by addition of $10 \mathrm{~mL}$ nitric acid (98\%, Sigma-Aldrich) to this solution. Subsequently, the mixture was stirred, until a white precipitate was observed, and then heated at $80^{\circ} \mathrm{C}$ for $5 \mathrm{~h}$ in an oil bath to obtain a transparent sol. The previously prepared $\mathrm{N}-\mathrm{TiO}_{2}$ powder $(2 \mathrm{~g})$ was added to this sol, after which this mixture was sonicated for $1 \mathrm{~h}$ to obtain the final coating solution. The previously cleaned GF was dipped into the coating solution $(500 \mathrm{~mL})$ in a home-made glass container $(12 \mathrm{~cm} \times 23 \mathrm{~cm} \times 2 \mathrm{~cm})$ for $10 \mathrm{~min}$, taken out slowly, and dried at room temperature for $2 \mathrm{~h}$. These dip-coating and drying processes were conducted in triplicate to give high coating amounts. GF-N-TiO 2 powders having $\mathrm{N}$-to-Ti ratios of 0.06 , $0.08,0.10$, and 0.12 were named as $\mathrm{GF}-\mathrm{N}-\mathrm{TiO}_{2}-0.06$, GF$\mathrm{N}-\mathrm{TiO}_{2}-0.08, \mathrm{GF}-\mathrm{N}-\mathrm{TiO}_{2}-0.10$, and GF-N-TiO $2-0.12$, respectively. In addition, a reference photocatalyst, $\mathrm{GF}^{-\mathrm{TiO}_{2}}$, was prepared using P25 $\mathrm{TiO}_{2}$ instead of $\mathrm{N}-\mathrm{TiO}_{2}$, following the same coating procedure as used for the preparation of GF$\mathrm{N}-\mathrm{TiO}_{2}$. The characteristics of as-prepared GF-N-TiO, GF$\mathrm{TiO}_{2}$, and pretreated GF were determined using scanning electron microscopy (SEM, Hitachi S-4300 FE-SEM), energydispersive X-ray spectroscopy (EDX, Hitachi EDX-350), $\mathrm{X}$-ray diffraction spectroscopy (XRD, Rigaku D/max-2500 diffractometer), and UV-visible spectroscopy (Varian CARY $5 \mathrm{G})$. For the EDX analysis, the sample was pretreated using a Pt-coating system (Hitachi E-1030).

2.2. Determination of Aromatic VOC Degradation Efficiencies. The degradation efficiencies of aromatic VOCs were 
investigated using GF-N-TiO 2 , GF- $\mathrm{TiO}_{2}$, and GF, which were placed in a cylindrical photocatalytic reactor. The reactor was prepared using a Pyrex tube (a length of $26.5 \mathrm{~cm}$ and an inner diameter of $3.8 \mathrm{~cm}$ ), where a cylindrical-shaped daylight lamp (F8T5DL, Youngwha Lamp Co.) with a spectral range of 400$720 \mathrm{~nm}$ was inserted. The light intensities provided by the daylight lamp were $3.2 \mathrm{~mW} \mathrm{~cm}^{-2}$ at a distance from the light source to the surface of photocatalysts. The inner surface of the Pyrex tube was covered by a GF-Fe-TiO, $\mathrm{GF}_{2}-\mathrm{TiO}_{2}$, or GF sheet. Zero-grade dried air was supplied by an air cylinder and was allowed to flow through a carbon filter for repurification. The dried air stream flowed through two water-containing impingers in tandem, which were partially immersed in a water bath, for air humidification. The relative humidity $(\mathrm{RH})$ levels were adjusted by controlling the mixing ratio of dried with humidified air flows. Standard compounds were prepared by adjusting the mixing ratio of the humidified air with TEX prepared by vaporizing liquid standards, which were injected into a heated glass chamber via a syringe pump (KDS 210, KD Scientific). Lastly, the prepared standard compounds were transferred to the reactor for photocatalytic decomposition efficiency tests. The degradation efficiency tests were conducted under different operational conditions by controlling two important factors: air flow rate (AFR: 1.0, 2.0, 3.0, and $4.0 \mathrm{~L} \mathrm{~min}^{-1}$ ) and initial concentration (IC: $0.1,0.5,1.0$, and $2.0 \mathrm{ppm}$ ). When evaluating a specific factor, the other factor was held constant at the following values: IC, $0.1 \mathrm{ppm}$, and AFR, $1.0 \mathrm{~L} \mathrm{~min}^{-1}$. In addition, the RH was fixed at $45 \%$, which represents a comfortable value for typical indoor life.

Air measurements were carried out at the inlet and outlet ports of the cylindrical reactor by drawing air through a stainless steel tubing trap containing Tenax GC adsorbent. Gaseous species collected in the adsorbent trap were analyzed using a gas chromatograph/mass spectrometer (Perkin Elmer Clarus SQ 8) (GC/MS) coupled to a thermal desorbing device (Perkin Elmer ATD 350). The gases were qualitatively analyzed based on both their retention times and mass spectra (Wiley 275 software library). Each chemical species was qualitatively analyzed based on calibration curves, which were determined using five concentrations standardized to an internal standard chemical. For the quality control program, a laboratory blank and spiked standard trap samples were analyzed. The entire experimental procedure was repeated three times to give more reliable data and the average values are presented in this paper.

\section{Results and Discussion}

3.1. Characteristics of As-Prepared Photocatalysts. The surface characteristics of a representative GF-N-TiO ${ }_{2}$ with a N-toTi ratio of $0.08\left(\right.$ GF- $\left.-\mathrm{NiO}_{2}-0.08\right)$ as well as a reference photocatalyst $\left(\mathrm{GF}-\mathrm{TiO}_{2}\right)$ were examined using SEM, EDX, XRD, and UV-visible absorption analyses. Based on the SEM micrographs (Figure 2), GF-N-TiO $2-0.08$ showed a better coating of $\mathrm{N}-\mathrm{TiO}_{2}$ on GF relative to $\mathrm{GF}^{-\mathrm{TiO}_{2}}$. The enhanced coating efficiency for GF-N-TiO -0.08 was ascribed to the strong agglomerating nature of $\mathrm{N}^{-\mathrm{TiO}_{2}}$ [17]. Consistently, previous researchers $[22,34]$ have found a higher aggregation
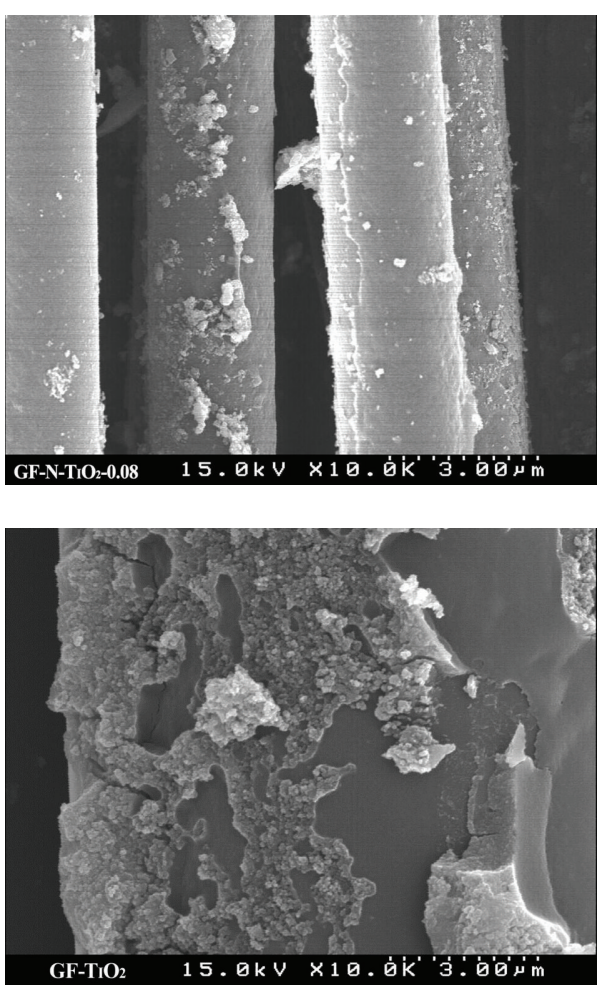

FIGURE 2: Scanning electron microscopy of GF-N-TiO 2 with a N-toTi ratio of $0.08($ GF-N-TiO $2-0.08)$ and $\mathrm{GF}-\mathrm{TiO}_{2}$.

property of $\mathrm{N}-\mathrm{TiO}_{2}$ as compared to pure $\mathrm{TiO}_{2}$ by SEM analysis.

As illustrated in Figure 3, the EDX spectrum of GF-N$\mathrm{TiO}_{2}-0.08$ showed peaks of $\mathrm{Ti}, \mathrm{O}$, and $\mathrm{N}$ atoms, whereas the spectrum of $\mathrm{GF}^{-} \mathrm{TiO}_{2}$ showed only $\mathrm{Ti}$ and $\mathrm{O}$ atom bands; those bands assigned to $\mathrm{Ti}$ and $\mathrm{O}$ atoms were associated with $\mathrm{TiO}_{2}$, while the $\mathrm{N}$ atom band was ascribed to $\mathrm{N}$ atoms embedded in the $\mathrm{TiO}_{2}$ lattice. Therefore, EDX spectra analysis demonstrated that $\mathrm{N}$ atoms could be successfully incorporated into $\mathrm{TiO}_{2}$ to allow the prepared photocatalyst be activated under visible-light irradiation conditions. In agreement with our results, Gurkan et al. [22] found the existence of $\mathrm{N}$ atoms for $\mathrm{N}_{-} \mathrm{TiO}_{2}$ synthesized using a wet incorporation process through EDX analysis. The undesignated peaks in the EDX spectra were attributed to Pt, which was used for sample coating prior to EDX analysis; no other evident impurities were observed in the samples.

Figure 4 illustrates the XRD spectra of GF-N-TiO ${ }_{2}-0.08$ and the reference $\mathrm{GF}-\mathrm{TiO}_{2}$ photocatalyst. Both photocatalysts displayed an anatase phase with a major band at $25.3^{\circ} 2 \theta$ and a rutile phase with a major band at $27.3^{\circ} 2 \theta$. These diffractograms were similar to those of Degussa P25 TiO reported in other studies $[22,35]$, indicating that $\mathrm{N}$ incorporated into $\mathrm{TiO}_{2}$ for the GF-N-TiO $2-0.08$ photocatalyst did not alter the crystal structures of $\mathrm{TiO}_{2}$. Gurkan et al. [22] also reported that the XRD patterns of $\mathrm{N}-\mathrm{TiO}_{2}$ were similar to those of pure $\mathrm{P} 25 \mathrm{TiO}_{2}$. However, any $\mathrm{N}$ dopant-associated bands were not found in the spectrum of GF-N-TiO -0.08 . These findings suggest that $\mathrm{N}$ atoms would not chemically 

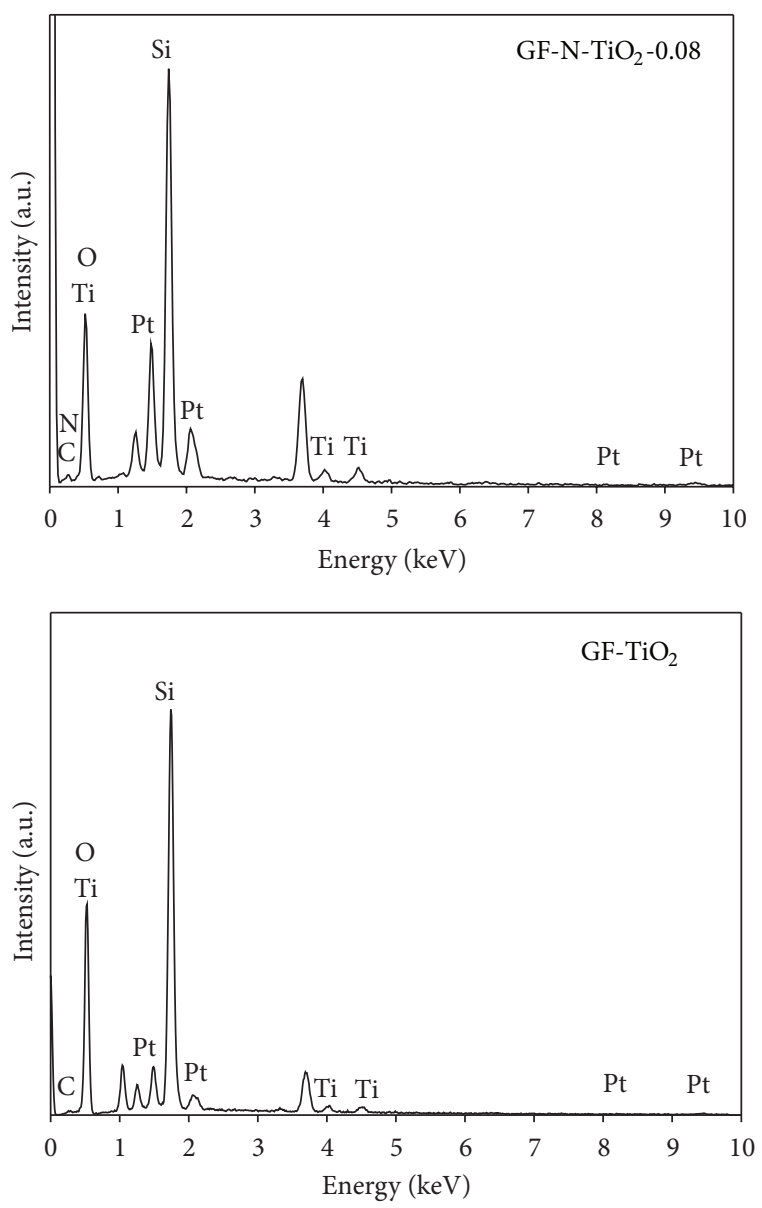

Figure 3: Energy-dispersive X-ray images of GF-N-TiO ${ }_{2}$ with a Nto-Ti ratio of $0.08\left(\mathrm{GF}-\mathrm{N}-\mathrm{TiO}_{2}-0.08\right)$ and $\mathrm{GF}-\mathrm{TiO}_{2}$.

react to produce new crystalline structures, such as $\mathrm{TiN}$, but are rather incorporated interstitially and substitutionally into the $\mathrm{TiO}_{2}$ structure [15]. Otherwise, low amounts of $\mathrm{N}$ atoms impregnated into $\mathrm{TiO}_{2}$ in GF-N-TiO $2-0.08$ could not be detected by XRD possibly due to its limited measurement sensitivity.

The sizes of crystal phases of the GF-N-TiO 2 photocatalysts and reference $\mathrm{GF}^{-\mathrm{TiO}_{2}}$ were estimated based on the Scherrer formula [36]. The anatase crystallite size of GF$\mathrm{N}-\mathrm{TiO}_{2}-0.08(36.61 \mathrm{~nm})$ was smaller than that of $\mathrm{GF}-\mathrm{TiO}_{2}$ $(39.31 \mathrm{~nm})$, while the crystallite sizes of GF-N-TiO $-0.06, \mathrm{GF}-$ $\mathrm{N}-\mathrm{TiO}_{2}-0.10$, and $\mathrm{GF}-\mathrm{N}-\mathrm{TiO}_{2}-0.12$ were $36.49,36.72$, and $37.04 \mathrm{~nm}$, respectively. Gurkan et al. [22] also reported a smaller crystallite size for $\mathrm{N}-\mathrm{TiO}_{2}$ powder relative to that of pure $\mathrm{TiO}_{2}$ powder. The reduction in the crystallite size observed for $\mathrm{GF}-\mathrm{N}-\mathrm{TiO}_{2}-0.08$ was ascribed to the formation of compressive strain in the crystallite structure, which would likely be due to the transformation of electronic structure resulting from $\mathrm{N}$ dopant ion incorporation into $\mathrm{TiO}_{2}$ [22].

Figure 5 shows the UV-visible spectra of the GF-N-TiO photocatalysts and reference $\mathrm{GF}_{-}-\mathrm{TiO}_{2}$. The reference GF$\mathrm{TiO}_{2}$ exhibited an absorption edge at a wavelength of around $420 \mathrm{~nm}$, which was in good agreement with that reported in previous studies $[22,34]$. In contrast, the UV-visible spectra

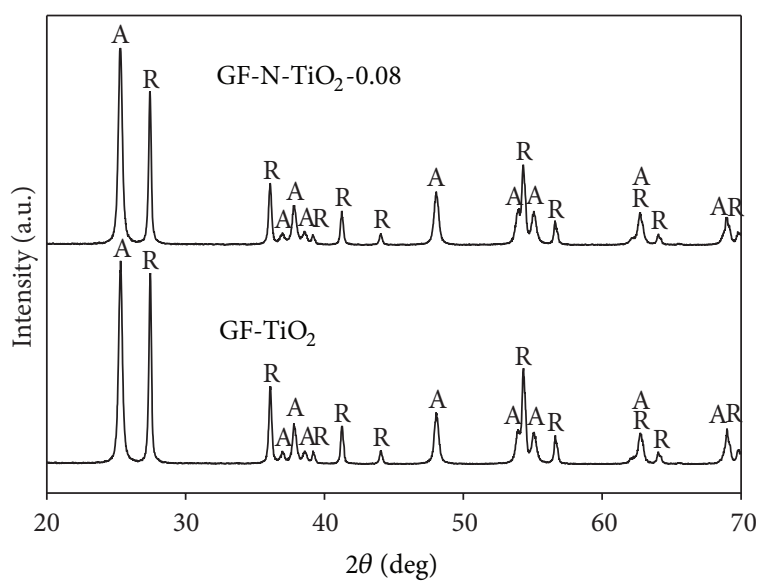

\section{A: Anatase R: Rutile}

FIgUre 4: X-ray diffraction patterns of GF-N-TiO 2 with a N-to-Ti ratio of $0.08\left(\mathrm{GF}-\mathrm{N}-\mathrm{TiO}_{2}-0.08\right)$ and $\mathrm{GF}-\mathrm{TiO}_{2}$.

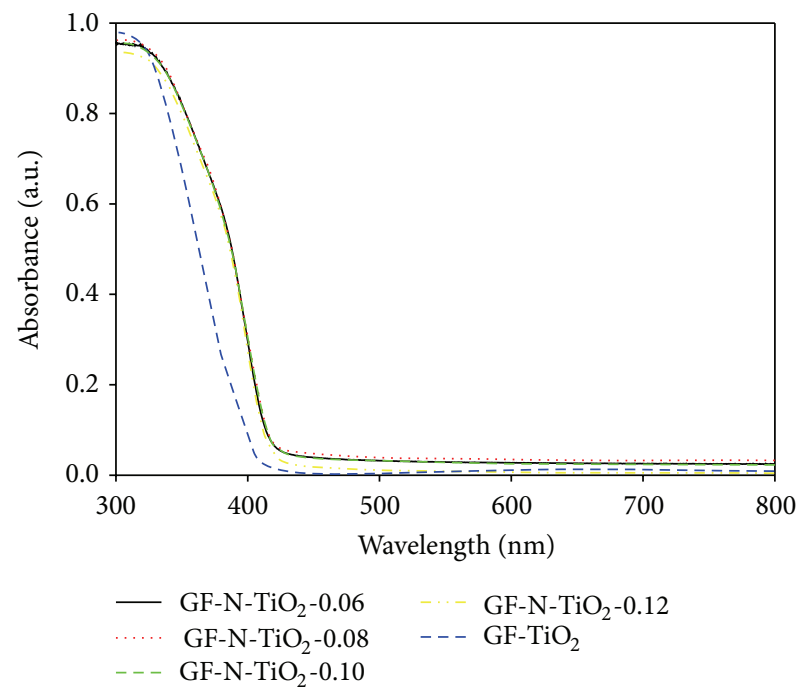

Figure 5: UV-visible spectra of GF-N-TiO -0.06 , GF-N-TiO -0.08 , GF-N-TiO $2-0.10$, GF-N-TiO -0.12 , and $\mathrm{GF}_{2}-\mathrm{TiO}_{2}$.

of GF-N-TiO 2 photocatalysts were highly shifted toward the visible region, which was consistent with the results reported by Gurkan et al. [22]. These shifts toward the visible region of the spectrum were attributed to the generation of a narrowed band gap level, which is located between the valence and conduction bands of $\mathrm{TiO}_{2}$. Overall, it was suggested that the prepared GF-N-TiO 2 photocatalysts could be functional under visible-light irradiation as well.

3.2. Photocatalytic Degradation of Aromatic VOCs. The photocatalytic degradation of TEX was conducted using the prepared GF-N-TiO 2 photocatalysts with different N-to-Ti ratios and $\mathrm{GF}_{-} \mathrm{TiO}_{2}$ under visible-light irradiation following threehour adsorption process. As shown in Figure 6, a control photolysis test performed using an uncoated GF displayed no degradation of TEX at all under visible-light irradiation, 


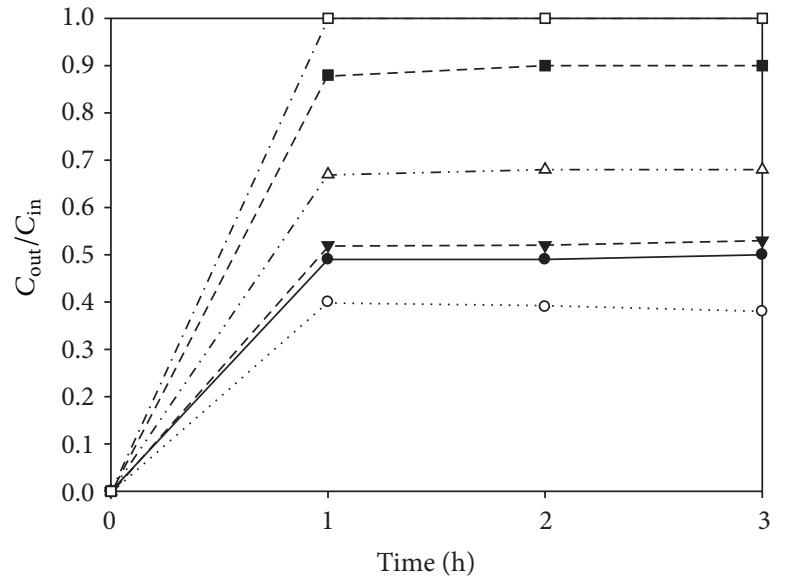

(a)

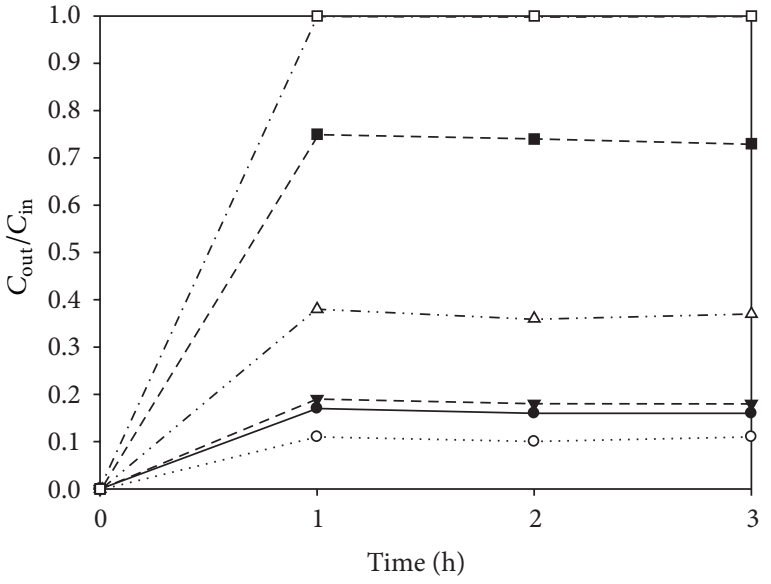

(b)

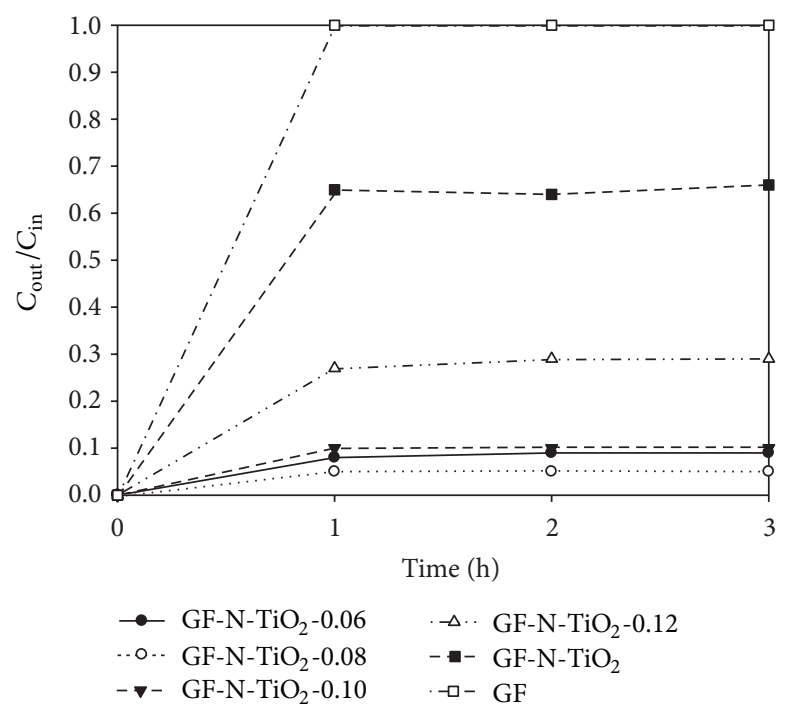

(c)

Figure 6: Time-series ratios $\left(C_{\text {out }} / C_{\text {in }}\right)$ of outlet-to-inlet concentrations of (a) toluene, (b) ethyl benzene, and (c) o-xylene as obtained using GF-N-TiO ${ }_{2}$ photocatalysts with different N-to-Ti ratios $\left(\mathrm{GF}_{-\mathrm{N}}-\mathrm{TiO}_{2}-0.06, \mathrm{GF}-\mathrm{N}-\mathrm{TiO}_{2}-0.08, \mathrm{GF}-\mathrm{N}-\mathrm{TiO}_{2}-0.10\right.$, and GF-N-TiO -0.12 ), GF$\mathrm{TiO}_{2}$, and GF.

which was demonstrated by similar values for inlet and outlet concentrations of TEX. In addition, Figure 5 shows the outlet to inlet concentrations of TEX determined for four GF-N$\mathrm{TiO}_{2}$ photocatalysts $\left(\mathrm{GF}-\mathrm{N}-\mathrm{TiO}_{2}-0.06, \mathrm{GF}-\mathrm{N}-\mathrm{TiO}_{2}-0.08\right.$, GF$\mathrm{N}-\mathrm{TiO}_{2}-0.10$, and $\mathrm{GF}-\mathrm{N}-\mathrm{TiO}_{2}-0.12$ ) and the reference GF$\mathrm{TiO}_{2}$ photocatalyst over three-hour photocatalytic process. The outlet-to-inlet concentration ratios of the three target pollutants obtained using the GF-N-TiO 2 photocatalysts were lower than that of the $\mathrm{GF}^{-} \mathrm{TiO}_{2}$ photocatalyst, indicating that GF-N-TiO 2 photocatalysts had higher photocatalytic activity toward the degradation of TEX. For example, the average outlet-to-inlet concentration ratios of TEX for GF-N-TiO ${ }_{2}$ 0.06 were $0.51,0.18$, and 0.09 , respectively, while those of the $\mathrm{GF}^{-T i O} \mathrm{O}_{2}$ photocatalyst were $0.90,0.76$, and 0.67 , respectively. Similarly, Gurkan et al. [22] demonstrated a higher photocatalytic activity for the degradation of cefazolin in the aqueous phase relative to undoped $\mathrm{TiO}_{2}$ under sunlight irradiation.
Jo and Kim [21] also reported that daylight lamp-irradiated $\mathrm{N}-\mathrm{TiO}_{2}$, which was coated directly onto the inner wall of a Pyrex tubing reactor, showed superior performance for the degradation of aromatic VOCs compared to unmodified $\mathrm{TiO}_{2}$. The superior photocatalytic activity of the GF-N-TiO photocatalysts under visible-light irradiation was ascribed to their predisposition for visible-light absorbance owing to large specific surface area and the reduced impurity level localized in the top of valence band, as demonstrated from their UV-visible spectra (Figure 5), since the photocatalytic reaction rates are proportional to the amount of photon absorption by the photocatalysts $[6,37-40]$. The smaller crystallite size of GF-N-TiO 2 photocatalysts relative to the $\mathrm{GF}^{-\mathrm{TiO}_{2}}$ photocatalyst would also enhance their photocatalytic activity. Consequently, it was suggested that GF-N-TiO photocatalysts with visible-light activation can be applied to effectively degrade aromatic VOCs. 


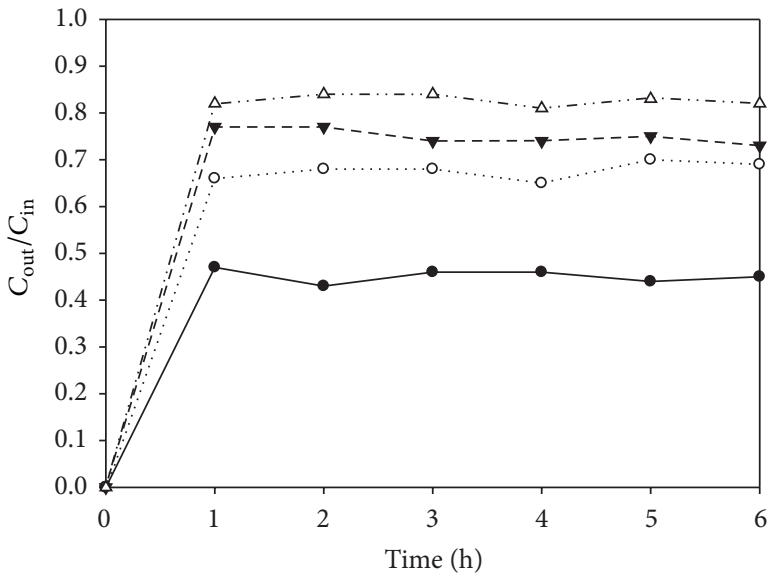

(a)

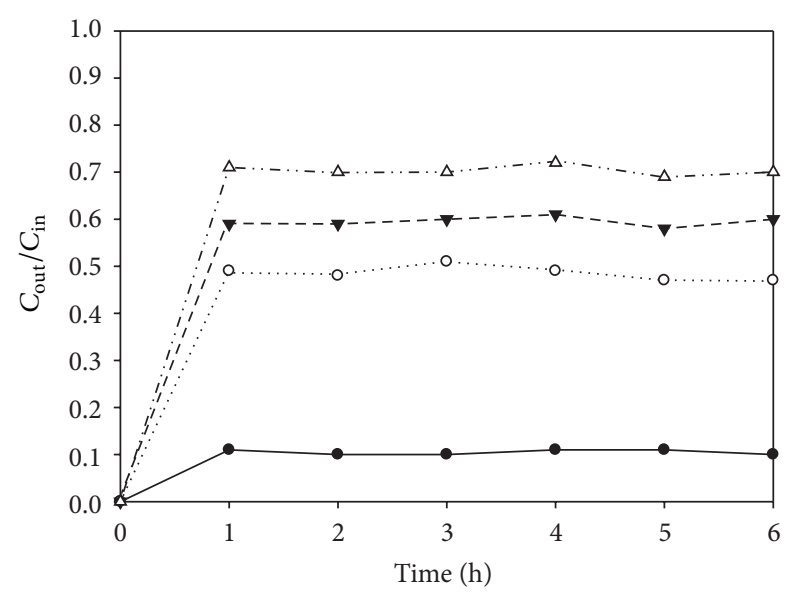

(b)

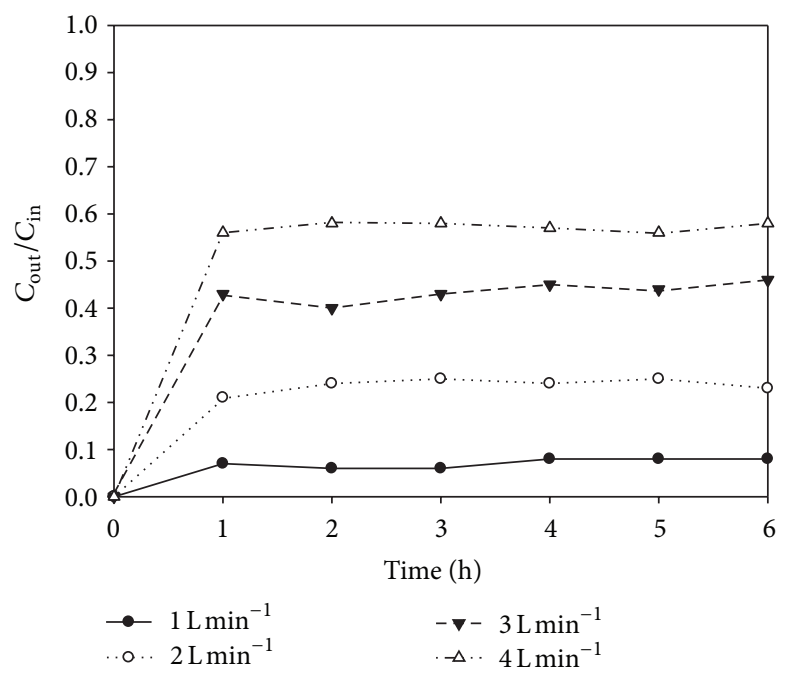

(c)

Figure 7: Time-series ratios $\left(C_{\text {out }} / C_{\text {in }}\right)$ of outlet-to-inlet concentrations of (a) toluene, (b) ethyl benzene, and (c) o-xylene as obtained using GF-N-TiO ${ }_{2}-0.08$ according to air-stream flow rates.

As also demonstrated in Figure 6, the photocatalytic activity of the GF-N-TiO 2 photocatalyst increased as the Nto-Ti ratio increased from 0.06 to 0.08 , but then decreased gradually as the N-to-Ti ratio increased further to 0.12 . These results indicate that there is an optimal $\mathrm{N}$-to-Ti ratio range for the synthesis of GF-N-TiO 2 photocatalysts. The decreasing pattern for high $\mathrm{N}$-to-Ti ratios was attributed to increased particle aggregation due to high amounts of $\mathrm{N}$ dopant, which results in the reduction in photocatalyst surface area [22]. The high amounts of $\mathrm{N}$ dopant might also decrease the separation distance of charge carriers, which result in an increase in the recombination of electron-hole pairs.

Figure 7 illustrates the outlet-to-inlet concentration ratios of TEX obtained using GF-N-TiO ${ }_{2}-0.08$ according to AFRs. The outlet-to-inlet concentration ratio of all the target compounds displayed an increasing trend as AFR increased, indicating that the photocatalytic activity of GF-N-TiO -0.08 decreased with increasing AFRs. Particularly, the average ratios of TEX increased from 0.47 to $0.82,0.11$ to 0.71 , and 0.09 to 0.59 , respectively, as the AFR increased from 1 to $4 \mathrm{~L} \mathrm{~min}^{-1}$. In agreement with our results, Jeong et al. [41] reported that the photocatalytic activity of Degussa P25 $\mathrm{TiO}_{2}$ for the degradation of benzene and toluene under UV irradiation showed a decreasing trend with increasing AFRs. These results might be ascribed to the mass transfer rate of the pollutant molecules from the air stream to the photocatalyst surface as well as photocatalytic reaction kinetics [42]. Unlike batch reactors, the mass transfer rates of gas-phase compounds in continuous-flow photocatalytic reactors are closely associated with the linear face velocities of the air stream [43]. In the present study, the linear face velocities increased as the AFR increased: the linear face velocities for AFRs of 1, 2, 3, and $4 \mathrm{~L} \mathrm{~min}^{-1}$ were $4.8,9.6,14.4$, and $19.2 \mathrm{~cm} \mathrm{~s}^{-1}$, respectively. These results suggested that the photocatalytic activity of the GF-N-TiO $2-0.08$ would increase as the AFR increased, owing to high mass-transfer rates; rather, the photocatalytic activity of GF-N-TiO -0.08 decreased with increasing AFR. As such, the low photocatalytic activity of GF-N-TiO $2-0.08$ at 


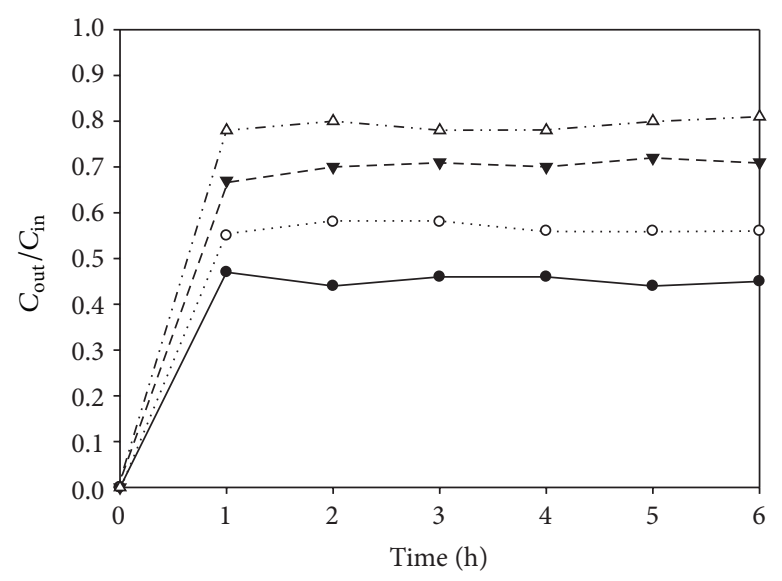

(a)

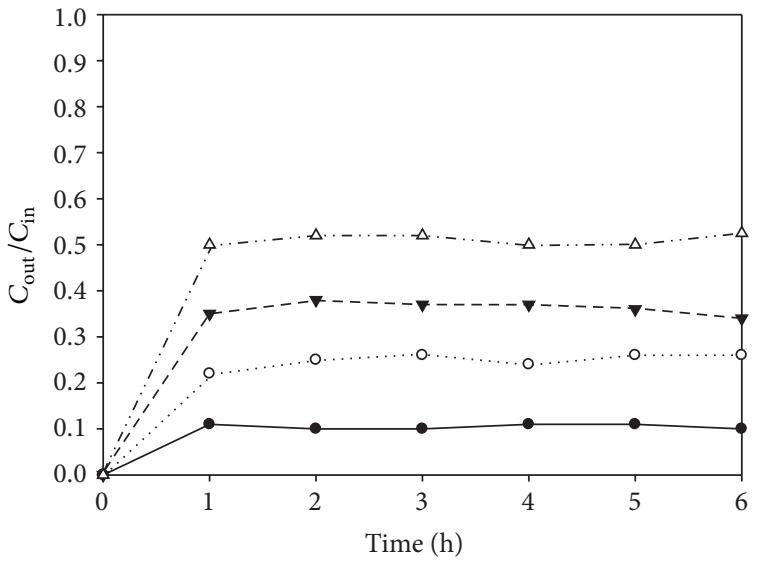

(b)

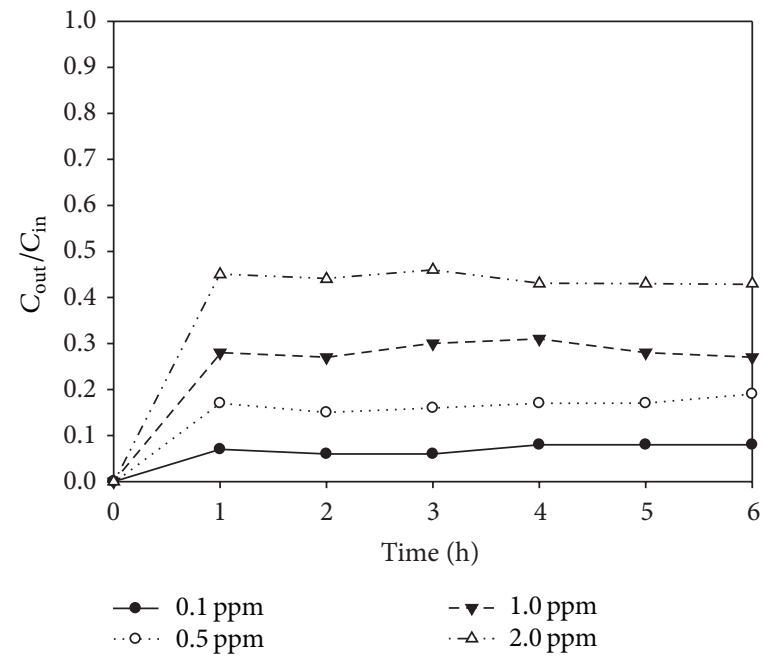

(c)

Figure 8: Time-series ratios $\left(C_{\text {out }} / C_{\text {in }}\right)$ of outlet-to-inlet concentrations of (a) toluene, (b) ethyl benzene, and (c) o-xylene as obtained using GF-N-TiO -0.08 according to inlet concentrations.

high AFRs was attributed to an insufficient reaction time in the photocatalytic reactor, suggesting that the photocatalytic activity of $\mathrm{GF}-\mathrm{N}-\mathrm{TiO}_{2}-0.08$ was likely limited by reaction kinetics on the photocatalyst surfaces. The reaction times for AFRs of $1,2,3$, and $4 \mathrm{~L} \mathrm{~min}^{-1}$, which were calculated by dividing the empty-space reactor volume by the AFRs, were $6.1,3.1,2.0$, and $1.5 \mathrm{~s}$, respectively.

Figure 8 shows the outlet-to-inlet concentration ratios of TEX obtained using GF-N-TiO -0.08 according to ICs. For all target compounds, the outlet-to-inlet concentration ratio increased as IC increased, indicating that the photocatalytic activity of GF-N-TiO -0.08 decreased with increasing ICs. At the lowest IC $(0.1 \mathrm{ppm})$, the average ratios of TEX were 0.41 , 0.10 , and 0.07 , respectively, whereas those at the highest IC $(2.0 \mathrm{ppm})$ were $0.79,0.49$, and 0.42 , respectively. Devahasdin et al. [44] also found that the photocatalytic degradation efficiencies of unmodified $\mathrm{TiO}_{2}$ for degradation of $\mathrm{NO}$ under $\mathrm{UV}$ irradiation decreased from $70-15 \%$ when IC was increased from 5-60 ppm. In addition, Jeong et al. [41] reported that the photocatalytic degradation efficiency of toluene determined using Degussa P25 $\mathrm{TiO}_{2}$ decreased gradually as IC increased from $0.6-10 \mathrm{ppm}$. The descending trend in photocatalytic activity with increasing IC was ascribed to competitive adsorption among TEX molecules on the catalyst surface; adsorption of chemical molecules onto the catalyst surface is known to be a crucial factor influencing the photocatalytic degradation of organic compounds [6].

\section{Conclusions}

This study investigated the applicability of glass fiber-based $\mathrm{N}$-doped titania $\left(\mathrm{GF}-\mathrm{N}-\mathrm{TiO}_{2}\right)$ under visible-light exposure for the photocatalytic degradation of aromatic VOCs. SEM results indicated the formation of aggregates for the GF$\mathrm{N}-\mathrm{TiO}_{2}$ photocatalyst, while EDX spectral analysis demonstrated that $\mathrm{N}$ elements were successfully incorporated into the $\mathrm{TiO}_{2}$ crystal lattice. The UV-visible spectra suggested that GF-N-TiO 2 photocatalysts could be functional under visible-light exposure. The prepared GF-N-TiO 2 photocatalysts showed superior photocatalytic performances to the 
reference $\mathrm{GF}-\mathrm{TiO}_{2}$ photocatalyst for degradation of aromatic VOCs under visible-light irradiation. The prepared GF-N$\mathrm{TiO}_{2}$ photocatalysts also showed a different dependence of $\mathrm{N}$ to-Ti ratios on photocatalytic degradation of aromatic VOCs, indicating that there is optimal range of $\mathrm{N}$-to-Ti ratios for the synthesis of GF-N-TiO 2 photocatalysts. In addition, AFR and IC were both found to be major influential factors on the photocatalytic performance of the GF-N-TiO ${ }_{2}$ photocatalysts. Overall, the GF-N-TiO 2 photocatalysts could be applied effectively for the degradation of aromatic VOCs under visible-light irradiation at optimal operation conditions.

\section{Conflict of Interests}

The authors declare that there is no conflict of interests regarding the publication of this paper.

\section{Acknowledgments}

This work was supported by the National Research Foundation of Korea (NRF) Grant funded by the Korea government (MEST) (no. 2011-0027916) and through the GCRC-SOP (no. 2011-0030013).

\section{References}

[1] M. Leuchner and B. Rappenglück, "VOC source-receptor relationships in Houston during TexAQS-II," Atmospheric Environment, vol. 44, no. 33, pp. 4056-4067, 2010.

[2] O. O. Hänninen, E. Lebret, V. Ilacqua et al., "Infiltration of ambient PM2.5 and levels of indoor generated non-ETS PM2.5 in residences of four European cities," Atmospheric Environment, vol. 38, no. 37, pp. 6411-6423, 2004.

[3] K.-D. Kwon, W.-K. Jo, H.-J. Lim, and W.-S. Jeong, "Characterization of emissions composition for selected household products available in Korea," Journal of Hazardous Materials, vol. 148, no. 1-2, pp. 192-198, 2007.

[4] S. H. Shin and W. K. Jo, "Longitudinal variations in indoor VOC concentrations after moving into new apartments and indoor source characterization," Environmental Science and Pollution Research, vol. 20, pp. 3696-3707, 2013.

[5] IARC (International Agency for Research on Cancer), "List of classifications by alphabetical order," 2013, http://monographs .iarc.fr/ENG/Classification/index.php.

[6] M. A. Henderson, "A surface science perspective on $\mathrm{TiO}_{2}$ photocatalysis," Surface Science Reports, vol. 66, no. 6-7, pp. 185297, 2011.

[7] T. Ohno, M. Akiyoshi, T. Umebayashi, K. Asai, T. Mitsui, and M. Matsumura, "Preparation of S-doped $\mathrm{TiO}_{2}$ photocatalysts and their photocatalytic activities under visible light," Applied Catalysis A: General, vol. 265, no. 1, pp. 115-121, 2004.

[8] C.-C. Hu, T.-C. Hsu, and S.-Y. Lu, "Effect of nitrogen doping on the microstructure and visible light photocatalysis of titanate nanotubes by a facile cohydrothermal synthesis via urea treatment," Applied Surface Science, vol. 280, pp. 171-178, 2013.

[9] H. Guo, M. Kemell, M. Heikkilä, and M. Leskelä, "Noble metalmodified $\mathrm{TiO}_{2}$ thin film photocatalyst on porous steel fiber support," Applied Catalysis B: Environmental, vol. 95, no. 3-4, pp. 358-364, 2010.
[10] S. Kment, H. Kmentova, P. Kluson et al., "Notes on the photoinduced characteristics of transition metal-doped and undoped titanium dioxide thin films," Journal of Colloid and Interface Science, vol. 348, no. 1, pp. 198-205, 2010.

[11] X. Chen, W. Wang, H. Xiao et al., "Accelerated $\mathrm{TiO}_{2}$ photocatalytic degradation of Acid Orange 7 under visible light mediated by Peroxymonosulfate," Chemical Engineering Journal, vol. 193194, pp. 290-295, 2012.

[12] Y. Wang, J. Zhang, L. Liu, C. Zhu, X. Liu, and Q. Su, "Visible light photocatalysis of $\mathrm{V}_{2} \mathrm{O}_{5} / \mathrm{TiO}_{2}$ nanoheterostructures prepared via electrospinning," Materials Letters, vol. 75, pp. 95-98, 2012.

[13] W.-K. Jo, "Coupling of titania with multiwall carbon nanotubes for decomposition of gas-phase pollutants under simulated indoor conditions," Journal of Air \& Waste Management Association, vol. 63, pp. 963-970, 2013.

[14] W.-K. Jo and H.-J. Kang, "Titanium dioxide-graphene oxide composites with different ratios supported by Pyrex tube for photocatalysis of toxic aromatic vapors," Powder Technology, vol. 250, pp. 115-121, 2013.

[15] R. Asahi, T. Morikawa, T. Ohwaki, K. Aoki, and Y. Taga, "Visible-light photocatalysis in nitrogen-doped titanium oxides," Science, vol. 293, no. 5528, pp. 269-271, 2001.

[16] M. Pelaez, N. T. Nolan, S. C. Pillai et al., "A review on the visible light active titanium dioxide photocatalysts for environmental applications," Applied Catalysis B: Environmental, vol. 125, pp. 331-349, 2012.

[17] C. di Valentin, E. Finazzi, G. Pacchioni et al., "N-doped $\mathrm{TiO}_{2}$ : theory and experiment," Chemical Physics, vol. 339, no. 1-3, pp. 44-56, 2007.

[18] C. di Valentin, G. Pacchioni, A. Selloni, S. Livraghi, and E. Giamello, "Characterization of paramagnetic species in Ndoped $\mathrm{TiO}_{2}$ powders by EPR spectroscopy and DFT calculations," Journal of Physical Chemistry B, vol.109, no. 23, pp. 1141411419, 2005.

[19] N. Serpone, "Is the band gap of pristine $\mathrm{TiO}_{2}$ narrowed by anion- and cation-doping of titanium dioxide in second-generation photocatalysts?" Journal of Physical Chemistry B, vol. 110, no. 48, pp. 24287-24293, 2006.

[20] A. V. Emeline, V. N. Kuznetsow, V. K. Rybchuk, and N. Serpne, "Visible-light-active titania photocatalysts: the case of N-doped $\mathrm{TiO}_{2} \mathrm{~s}$ - properties and some fundamental issues," International Journal of Photoenergy, vol. 2008, Article ID 258394, 19 pages, 2008.

[21] W.-K. Jo and J.-T. Kim, "Application of visible-light photocatalysis with nitrogen-doped or unmodified titanium dioxide for control of indoor-level volatile organic compounds," Journal of Hazardous Materials, vol. 164, no. 1, pp. 360-366, 2009.

[22] Y. Y. Gurkan, N. Turkten, A. Hatipoglu, and Z. Cinar, "Photocatalytic degradation of cefazolin over $\mathrm{N}$-doped $\mathrm{TiO}_{2}$ under $\mathrm{UV}$ and sunlight irradiation: prediction of the reaction paths via conceptual DFT,' Chemical Engineering Journal, vol. 184, pp. 113-124, 2012.

[23] A. Y. Shan, T. I. M. Ghazi, and S. A. Rashid, "Immobilisation of titanium dioxide onto supporting materials in heterogeneous photocatalysis: a review," Applied Catalysis A: General, vol. 389, no. 1-2, pp. 1-8, 2010.

[24] C. L. Bianchi, C. Pirola, E. Selli, and S. Biella, "Photocatalytic $\mathrm{NO}_{x}$ abatement: the role of the material supporting the $\mathrm{TiO}_{2}$ active layer," Journal of Hazardous Materials, vol. 211-212, pp. 203-207, 2012. 
[25] J. Matos, E. García-López, L. Palmisano, A. García, and G. Marcì, "Influence of activated carbon in $\mathrm{TiO}_{2}$ and $\mathrm{ZnO}$ mediated photo-assisted degradation of 2-propanol in gas-solid regime," Applied Catalysis B: Environmental, vol. 99, pp. 170-180, 2010.

[26] W. K. Jo, S. H. Shin, and E. S. Hwang, "Removal of dimethyl sulfide utilizing activated carbon fiber-supported photocatalyst in continuous-flow system," Journal of Hazardous Materials, vol. 191, no. 1-3, pp. 234-239, 2011.

[27] J.-W. Shi, H.-J. Cui, J.-W. Chen et al., " $\mathrm{TiO}_{2} /$ activated carbon fibers photocatalyst: effect of coating procedures on the microstructure, adhesion property, and photocatalytic ability," Journal of Colloid and Interface Science, vol. 388, pp. 201-208, 2012.

[28] T. E. Agustina, H. M. Ang, and V. K. Pareek, "Treatment of winery wastewater using a photocatalytic/photolytic reactor," Chemical Engineering Journal, vol. 135, no. 1-2, pp. 151-156, 2008.

[29] S. W. Verbruggen, S. Ribbens, T. Tytgat et al., "The benefit of glass bead supports for efficient gas phase photocatalysis: case study of a commercial and a synthesised photocatalyst," Chemical Engineering Journal, vol. 174, no. 1, pp. 318-325, 2011.

[30] A. K. Alves, F. A. Berutti, F. J. Clemens, T. Graule, and C. P. Bergmann, "Photocatalytic activity of titania fibers obtained by electrospinning," Materials Research Bulletin, vol. 44, no. 2, pp. 312-317, 2009.

[31] C. Prahsarn, W. Klinsukhon, and N. Roungpaisan, "Electrospinning of PAN/DMF/H2O containing $\mathrm{TiO}_{2}$ and photocatalytic activity of their webs," Materials Letters, vol. 65, no. 15-16, pp. 2498-2501, 2011.

[32] A. Panniello, M. L. Curri, D. Diso et al., "Nanocrystalline $\mathrm{TiO}_{2}$ based films onto fibers for photocatalytic degradation of organic dye in aqueous solution," Applied Catalysis B: Environmental, vol. 121-122, pp. 190-197, 2012.

[33] S. Lin, X. Zhang, Q. Sun, T. Zhou, and J. Lu, "Fabrication of solar light induced $\mathrm{Fe}-\mathrm{TiO}_{2}$ immobilized on glass-fiber and application for phenol photocatalytic degradation," Materials Research Bulletin, vol. 48, pp. 4570-4575, 2013.

[34] J. Senthilnathan and L. Philip, "Photocatalytic degradation of lindane under UV and visible light using $\mathrm{N}$-doped $\mathrm{TiO}_{2}$," Chemical Engineering Journal, vol. 161, no. 1-2, pp. 83-92, 2010.

[35] M. M. Joshi, N. K. Labhsetwar, D. V. Parwate, and S. S. Rayalu, "Efficient photocatalytic hydrogen generation by silica supported and platinum promoted titanium dioxide," Materials Research Bulletin, vol. 48, pp. 3545-3552, 2013.

[36] B. D. Cullity and S. R. Stock, Elements of X-Ray Diffraction, Prentice Hall, Upper Saddle River, NJ, USA, 3rd edition, 2001.

[37] Q. Xiang, J. Yu, and M. Jaroniec, "Nitrogen and sulfur co-doped $\mathrm{TiO}_{2}$ nanosheets with exposed $\{001\}$ facets: synthesis, characterization and visible-light photocatalytic activity," Physical Chemistry Chemical Physics, vol. 13, no. 11, pp. 4853-4861, 2011.

[38] Q. Xiang, J. Yu, W. Wang, and M. Jaroniec, "Nitrogen self-doped nanosized $\mathrm{TiO}_{2}$ sheets with exposed $\{001\}$ facets for enhanced visible-light photocatalytic activity," Chemical Communications, vol. 47, no. 24, pp. 6906-6908, 2011.

[39] Z. Lin, A. Orlov, R. M. Lambert, and M. C. Payne, "New insights into the origin of visible light photocatalytic activity of nitrogendoped and oxygen-deficient anatase $\mathrm{TiO}_{2}$," Journal of Physical Chemistry B, vol. 109, no. 44, pp. 20948-20952, 2005.

[40] P. Zhou, J. Yu, and Y. Wang, "The new understanding on photocatalytic mechanism of visible-light response N-S codoped anatase $\mathrm{TiO}_{2}$ by first-principles," Applied Catalysis B: Environmental, vol. 142-143, pp. 45-53, 2013.
[41] J. Jeong, K. Sekiguchi, W. Lee, and K. Sakamoto, "Photodegradation of gaseous volatile organic compounds (VOCs) using $\mathrm{TiO}_{2}$ photoirradiated by an ozone-producing UV lamp: decomposition characteristics, identification of by-products and watersoluble organic intermediates," Journal of Photochemistry and Photobiology A: Chemistry, vol. 169, no. 3, pp. 279-287, 2005.

[42] K. Demeestere, J. Dewulf, and H. van Langenhove, "Heterogeneous photocatalysis as an advanced oxidation process for the abatement of chlorinated, monocyclic aromatic and sulfurous volatile organic compounds in air: state of the art," Critical Reviews in Environmental Science and Technology, vol. 37, no. 6, pp. 489-538, 2007.

[43] L. Yang, Z. Liu, J. Shi, H. Hu, and W. Shangguan, "Design consideration of photocatalytic oxidation reactors using $\mathrm{TiO}_{2}$ coated foam nickels for degrading indoor gaseous formaldehyde," Catalysis Today, vol. 126, no. 3-4, pp. 359-368, 2007.

[44] S. Devahasdin, C. Fan Jr., K. Li, and D. H. Chen, "TiO 2 photocatalytic oxidation of nitric oxide: transient behavior and reaction kinetics," Journal of Photochemistry and Photobiology A: Chemistry, vol. 156, no. 1-3, pp. 161-170, 2003. 

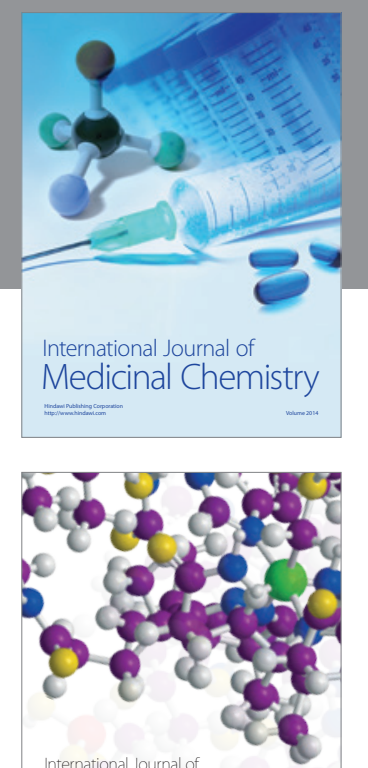

\section{Carbohydrate} Chemistry

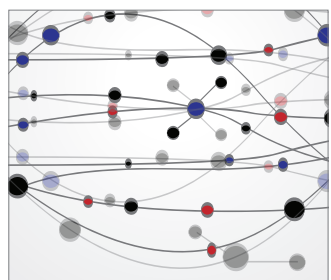

The Scientific World Journal
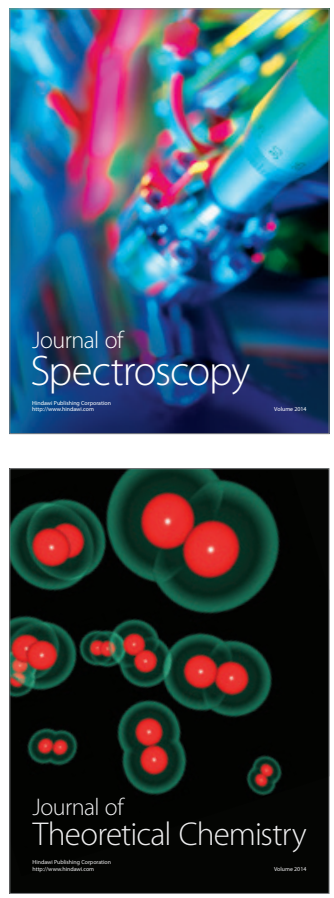
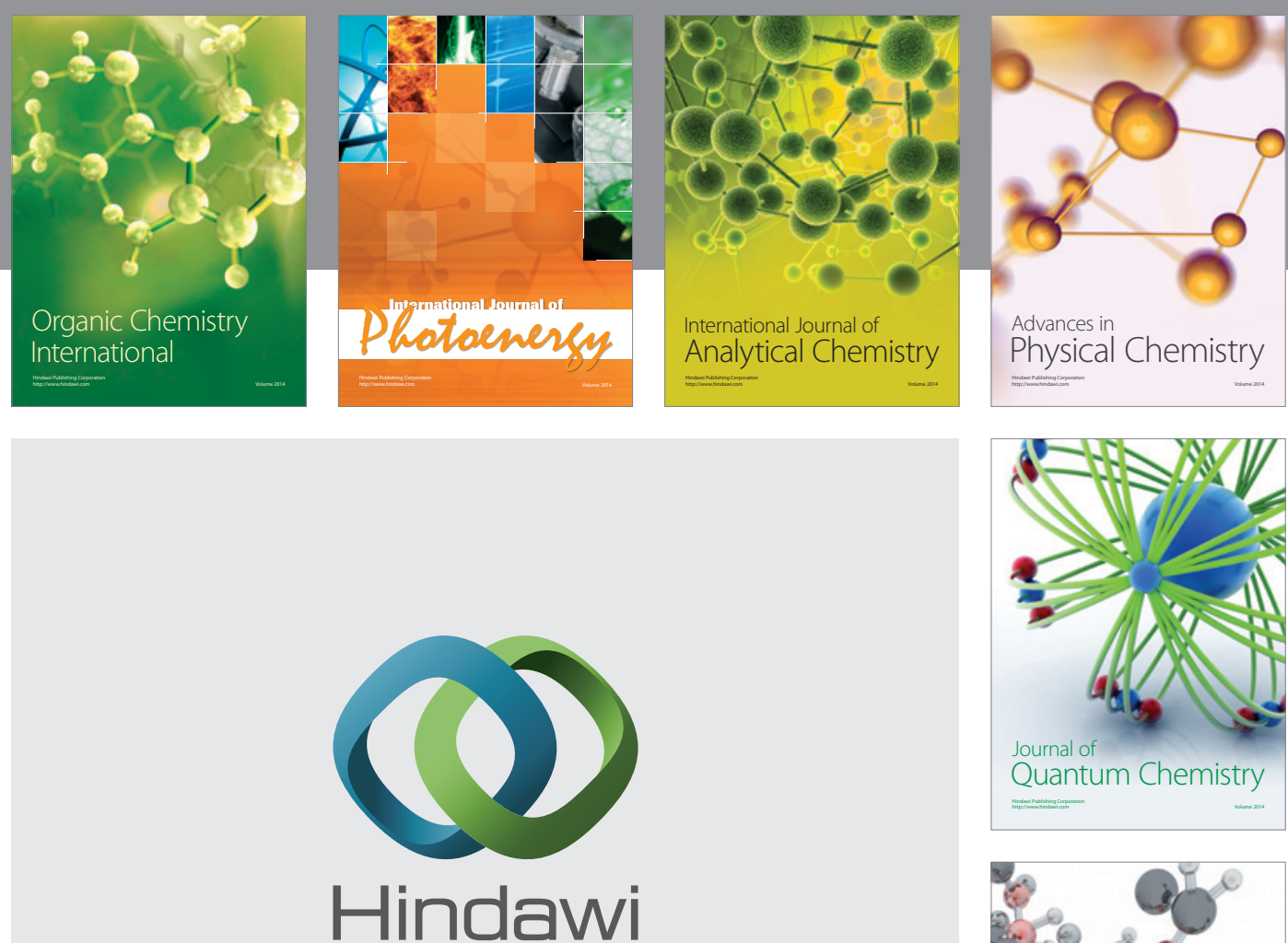

Submit your manuscripts at

http://www.hindawi.com

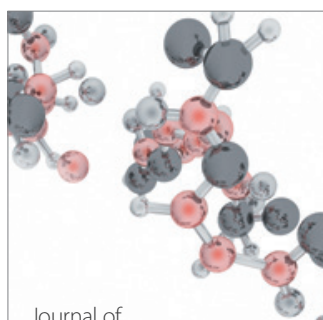

Analytical Methods

in Chemistry

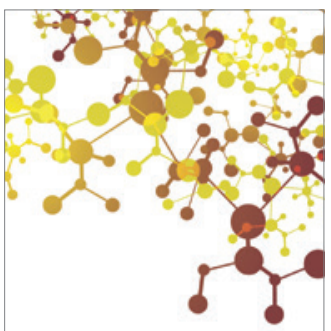

Journal of

Applied Chemistry

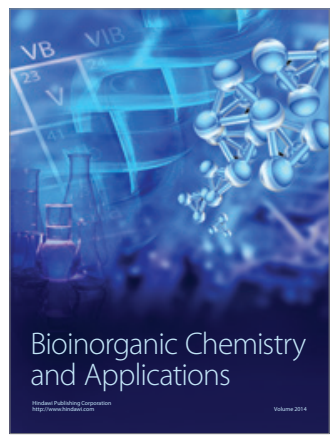

Inorganic Chemistry
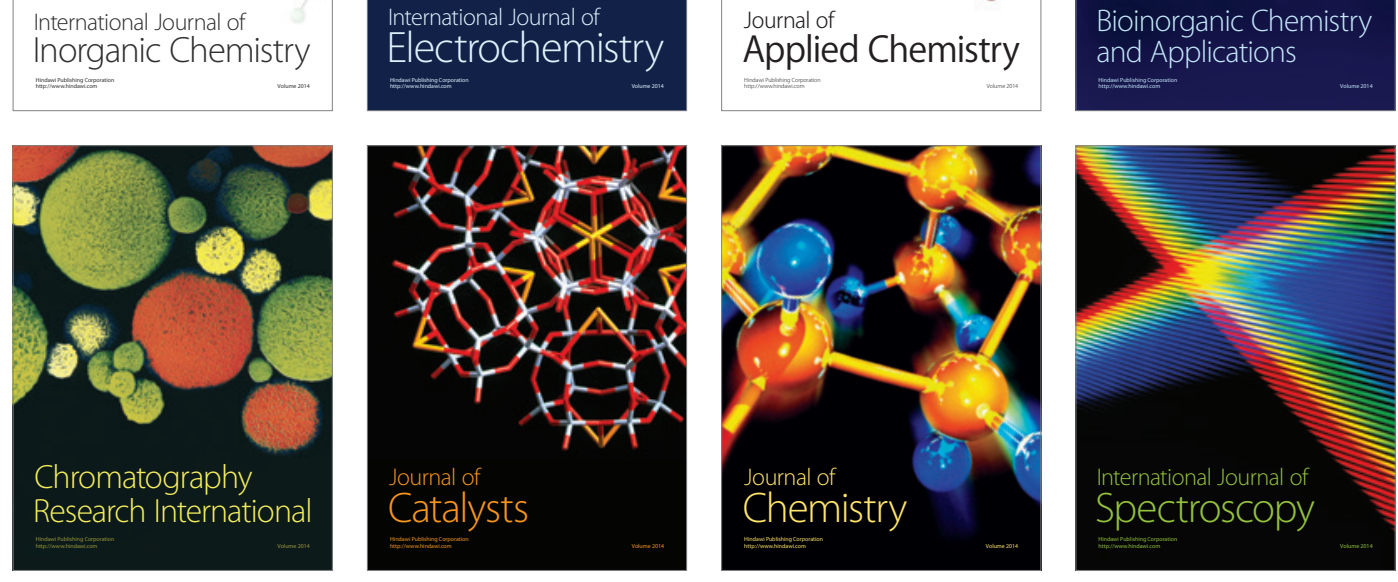